\title{
About the author
}

Dmitry Yu. Murzin studied Chemical Technology at the Mendeleev University of Chemical Technology in Moscow, Russia (1980-1986) and graduated with honors. He obtained his PhD (advisor Prof. M.I. Temkin) and DrSc degrees at Karpov Institute of Physical Chemistry, Moscow in 1989 and 1999, respectively. He worked at Universite Louis Pasteur, Strasbourg, France and Åbo Akademi University, Turku, Finland as a post-doc (1992-1994). In 1995-2000 he was associated with BASF, being involved in research, technical marketing and management. Since 2000 Prof. Murzin holds the Chair of Chemical Technology at Åbo Akademi University. He serves on the editorial boards of several journals in catalysis and chemical engineering field. He is an elected member of Academia Europaea and the Finnish Academy of Science and Letters.

Prof. Murzin is the coauthor with Prof. T. Salmi of a monograph Catalytic Kinetics (Elsevier, 2005, second edition in 2016), and author of Chemical Reaction Technology (de Gruyter, 2015) and Chemical Product Technology (de Gruyter, 2018). He holds several patents and is an author or coauthor of approximately 750 journal articles and book chapters. 
